15. Steckler, T. Drinkenburg W. H. Sahgal, A. \& Aggleton, J. P. Recognition memory in rats Il. Neuroanatomical substrates. Prog. Neurobiol. 54, 313-332 (1998).

16. Mumby, D. G., Gaskin, S., Glenn, M. J., Schramek, T. E. \& Lehmann, H. Hippocampal damage and exploratory preferences in rats: memory for objects, places, and contexts. Learn. Mem. 9, 49-57 (2002)

17. Eacott, M. J. \& Norman, G. Integrated memory for object, place, and context in rats: a possible model of episodic-like memory? J. Neurosci. 24, 1948-1953 (2004).

18. Macmillan, N. A. \& Creelman, C. D. Detection Theory: A User's Guide (Cambridge Univ. Press, New York, 1991).

19. Yonelinas, A. P., Kroll, N. E., Dobbins, I., Lazzara, M. \& Knight, R. T. Recollection and familiarity deficits in amnesia: convergence of remember-know, process dissociation, and receiver operating characteristic data. Neuropsychology 12, 323-339 (1998).

20. Yonelinas, A. P. Components of episodic memory: the contribution of recollection and familiarity. Phil. Trans. R. Soc. Lond. B 356, 1363-1374 (2001)

21. Hockley, W. E. Item versus associative information: further comparison of forgetting rates. J. Exp. Psychol. Learn. Mem. Cogn. 18, 1321-1330 (1992).

22. Yonelinas, A. P. \& Levy, B. J. Dissociating familiarity from recollection in human recognition memory: different rates of forgetting over short retention intervals. Psychon. Bull. Rev. 9, 575-582 (2002)

23. Murray, E. A. \& Mishkin, M. Object recognition and location memory in monkeys with excitotoxic lesions of the amygdala and hippocampus. J. Neurosci. 18, 6568-6582 (1998)

24. Tulving, E. Episodic memory: from mind to brain. Annu. Rev. Psychol. 53, 1-25 (2002).

25. Bunsey, M. \& Eichenbaum, H. Conservation of hippocampal memory function in rats and humans. Nature 379, 255-257 (1996)

26. Day, M., Langston, R. \& Morris, R. G. Glutamate-receptor-mediated encoding and retrieval of pairedassociate learning. Nature 424, 205-209 (2003).

27. Wood, E. R., Dudchenko, P. A., Robitsek, R. J. \& Eichenbaum, H. Hippocampal neurons encode information about different types of memory episodes occurring in the same location. Neuron 27, 623-633 (2000).

28. Kesner, R. P. in Neurobiology of Comparative Cognition (eds Kesner, R. P. \& Olton, D. S.) 179-203 (Lawrence Erlbaum, New Jersey, 1990).

29. Clark, R. E. \& Squire, L. R. Classical conditioning and brain systems: the role of awareness. Science $\mathbf{2 8 0}$ 77-81 (1998)

30. Fortin, N. J., Agster, K. L. \& Eichenbaum, H. Critical role of the hippocampus in memory for sequences of events. Nature Neurosci. 5, 458-462 (2002).

Supplementary Information accompanies the paper on www.nature.com/nature

Acknowledgements We thank J O'Connell, A. Milewski, L Giocomo, I. Estes, D. Tosa, R. Kline, J. Davidson and B. Goldberg for help with behavioural testing. We also thank R. Komorowski for histological work, A. Yonelinas for providing the least-squares curve-fitting algorithm and

J. Manns for comments on an earlier version of this manuscript. Supported by NIA and NIMH.

Competing interests statement The authors declare that they have no competing financial interests.

Correspondence and requests for materials should be addressed to H.B.E. (hbe@bu.edu)

\section{Restricted growth of Schwann cells lacking Cajal bands slows conduction in myelinated nerves}

Felipe A. Court, Diane L. Sherman, Thomas Pratt, Emer M. Garry, Richard R. Ribchester, David F. Cottrell, Susan M. Fleetwood-Walker \& Peter J. Brophy

Centre for Neuroscience Research, University of Edinburgh, Edinburgh EH9 1QH, UK

Nerve impulses are propagated at nodes of Ranvier in the myelinated nerves of vertebrates. Internodal distances have been proposed to affect the velocity of nerve impulse conduction $^{1}$; however, direct evidence is lacking, and the cellular mechanisms that might regulate the length of the myelinated segments are unknown. Ramón y Cajal described longitudinal and transverse bands of cytoplasm or trabeculae in internodal Schwann cells and suggested that they had a nutritive function ${ }^{2}$. Here we show that internodal growth in wild-type nerves is precisely matched to nerve extension, but disruption of the cytoplasmic bands in Periaxin-null mice impairs Schwann cell elongation during nerve growth. By contrast, myelination proceeds normally. The capacity of wild-type and mutant Schwann cells to elongate is cell-autonomous, indicating that passive stretching can account for the lengthening of the internode during limb growth. As predicted on theoretical grounds, decreased internodal distances strikingly decrease conduction velocities and so affect motor function. We propose that microtubule-based transport in the longitudinal bands of Cajal permits internodal Schwann cells to lengthen in response to axonal growth, thus ensuring rapid nerve impulse transmission.

Nodes of Ranvier in peripheral nerves are flanked by Schwann
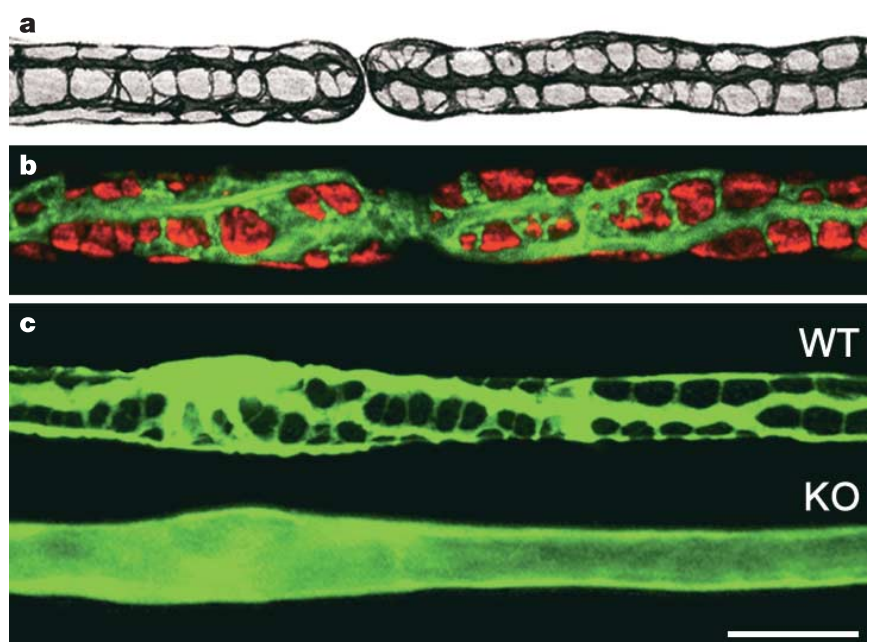
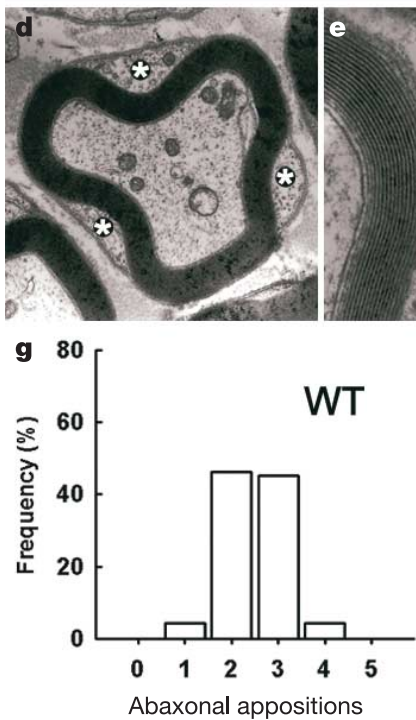
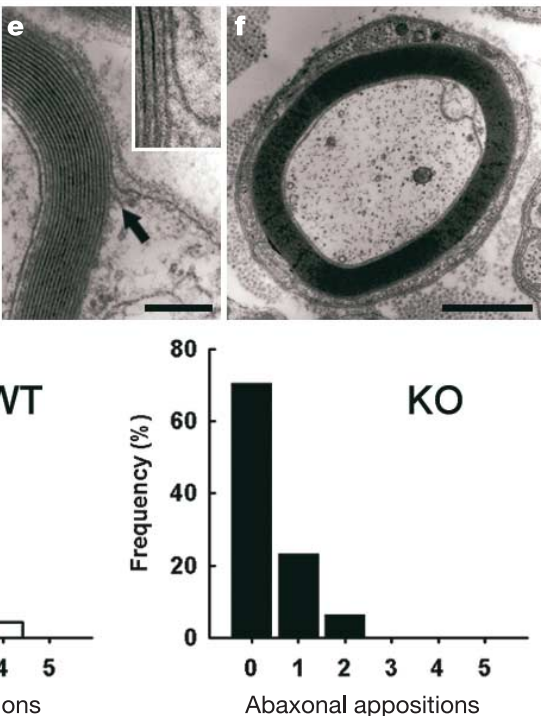

Figure 1 Longitudinal and transverse bands of Cajal in Schwann cells and their disruption in quadriceps nerve of Periaxin-null (KO) mice at 3 weeks. a, Longitudinal and transverse protoplasmic bands stained with silver by Ramón y Cajal (reproduced with permission) ${ }^{2}$. b. Teased fibres double-labelled with TRITC-phalloidin (green) and an antibody against DRP2 (red). Schwann cell cytoplasm is excluded from spheroidal clusters immunopositive for DRP2. c, Immunostaining of fibres from WT and Prx KO mice for the Schwann cell cytoplasmic protein $\mathrm{S} 100$. Scale bar, $20 \mu \mathrm{m}$ in $\mathbf{b}$ and $\mathbf{c}$. $\mathbf{d}-\mathbf{f}$, Electron micrographs of transverse sections of WT and KO quadriceps nerves. d, WT Schwann cell cytoplasm (asterisks) is restricted to regions delimited by appositions between the Schwann cell plasma membrane and the abaxonal layer of the myelin sheath. $\mathbf{e}$, The sharp transition between the apposition and cytoplasmic zones is shown by the arrow. Scale bar, $0.2 \mu \mathrm{m}$. The inset shows a high-power view of the transition zone. $\mathbf{f}$, In the absence of appositions in the KO, the Schwann cell cytoplasm forms a concentric ring around the myelin sheath. Scale bar, $1 \mu \mathrm{m}$ (d and $\mathbf{f}) . \mathbf{g}$, The proportion of abaxonal appositions per Schwann cell in WT and KO mice ( $n=3$ for WT and KO). 
cells, and in theory the length of the internodal Schwann cell should influence the rate of impulse conduction ${ }^{1}$. However, this has not been experimentally verified, largely because the two other main influences on conduction velocity, namely axon diameter and myelin thickness, normally co-vary with internodal length. Further, it is not known what mechanisms set the internodal distance and allow Schwann cells to elongate so extensively during nerve growth in vivo. The cytoplasmic meshwork beneath the plasma membrane of myelinating Schwann cells was first described by Ramón y Cajal (Fig. 1a), who speculated that these longitudinal and transverse protoplasmic trabeculae might have a nutritive function; however, their role in cell growth and maturation has remained unclear ${ }^{2}$.

We have previously identified spheroidal domains in the Schwann cell plasma membrane that contain the L-PeriaxinDystrophin-related protein 2 (DRP2)-Dystroglycan (PDG) complex $^{3}$. Double-labelling for DRP2 and cytoplasmic microfilaments by immunofluorescence showed that the PDG-rich domains are surrounded by cytoplasmic bands (Fig. 1b). To address the function of the bands, we first determined whether they were deranged in Periaxin-null $\left(\operatorname{Prx}{ }^{-/-}\right)$mice, because we had previously shown that the PDG complex is biochemically disrupted in these mice ${ }^{3}$. Immunostaining for the Schwann cell cytoplasmic marker S100 revealed that the cytoplasmic bands were absent from the Schwann cells of $\mathrm{Prx}^{-1-}$ mice (Fig. 1c). Electron microscopy showed that there were no appositions between the abaxonal surface of the myelin sheath and the plasma membrane of the $\operatorname{Prx}{ }^{-1-}$ Schwann cells (Fig. 1d-g), and this was consistent with our previous ultrastructural observation that DRP2 and Periaxin localize together at these appositions ${ }^{3}$. Instead of being compartmentalized in bands, the Schwann cell cytoplasm now formed a continuous annulus under the plasma membrane (Fig. 1f).

What are the consequences of disrupting the longitudinal Cajal bands, in the light of Ramón y Cajal's proposal that they might serve a nutritive function? Although inactivation of the Prx gene causes

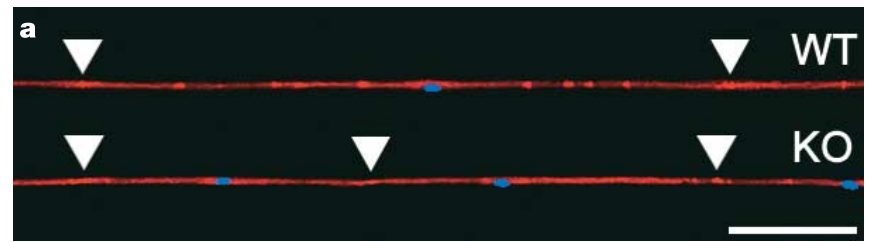

b

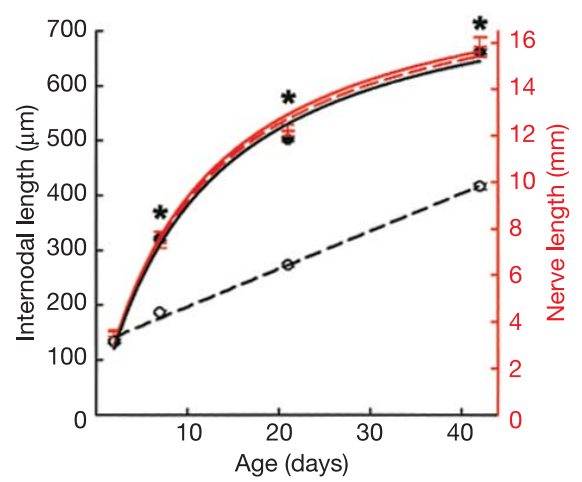

Figure 2 Internodal length is decreased in Schwann cells from $\mathrm{Prx}^{-1-}$ mice lacking Cajal bands. a, Teased fibres from 3-week-old WT and KO quadriceps nerves were stained with TRITC-phalloidin (red) and DAPI (blue). Nodes of Ranvier are indicated by arrowheads. Scale bar, $100 \mu \mathrm{m}$. b. The growth rate of WT and KO internodal lengths fitted a rectangular hyperbola and a straight line respectively. The growth rates of WT and KO nerve length were similar and both fitted rectangular hyperbolas (the null hypothesis that there was no significant difference between these fitted curves was supported by F-test; $P=0.945)$. Internodal lengths of WT (continuous black line) and KO (dashed black line) are comparable at 2 days. Thereafter, internodal growth of WT Schwann cells matches the increase in length of the quadriceps nerve (continuous red line) exactly. In contrast, the rate of internodal growth in the $\mathrm{KO}$ is decreased, even though the nerve grows at a normal rate (dashed red line). Values are means \pm s.e.m. for three animals (asterisks,

$P<0.0001$; Student's $t$-test for internodal lengths).

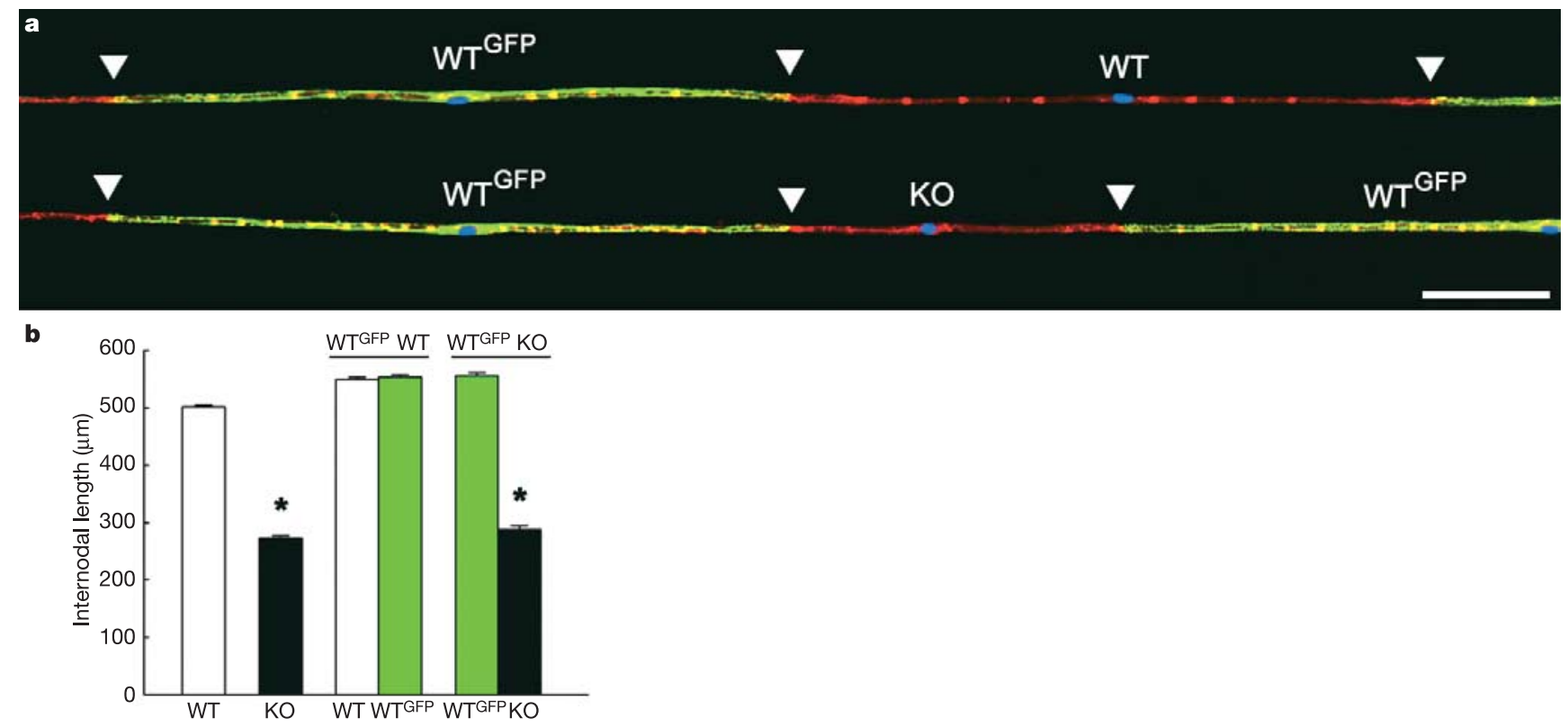

Figure 3 The capacity of Schwann cells to specify internodal length is cell-autonomous. Teased fibres in quadriceps nerves from 3-week-old mice were stained with

TRITC-phalloidin and DAPI. a, Nerves were from chimaeric mice containing GFP-tagged Schwann cells (S129 background) and either WT Schwann cells or Prx KO Schwann cells (both C57BL/6 background). The green signal is from GFP. Nodes of Ranvier are indicated by arrowheads. Scale bar, $100 \mu \mathrm{m}$. b. The first two bars show that internodal lengths from non-chimaeric $\mathrm{KO}$ mice are decreased ${ }^{*} P<0.0001$ by Student's $t$-test; $n=3$ ). In WT ${ }^{\mathrm{GFP}}$-WT chimaeras, both types of Schwann cells had similar internodal lengths. In
WT ${ }^{\text {GFP }}$-KO chimaeras, WT ${ }^{\text {GFP }}$ Schwann cells had similar internodal lengths to those in WT ${ }^{\text {GFP }}$-WT chimaeras, whereas KO Schwann cells had decreased internodal lengths (asterisks, $P<0.0001$ by Student's $t$-test; $n=3$ ). The internodes of Schwann cells in WT ${ }^{\text {GFP }}$-WT chimaeras were significantly larger than those of C57BL/6 WT $(P<0.0001$ by Student's $t$-test), but the internodal lengths of $K O$ Schwann cells were decreased similarly in a chimaeric environment and in a C57BL/ 6 environment $(P=0.07$ by Student's t-test). All values are means + s.e.m. 


\section{letters to nature}

a
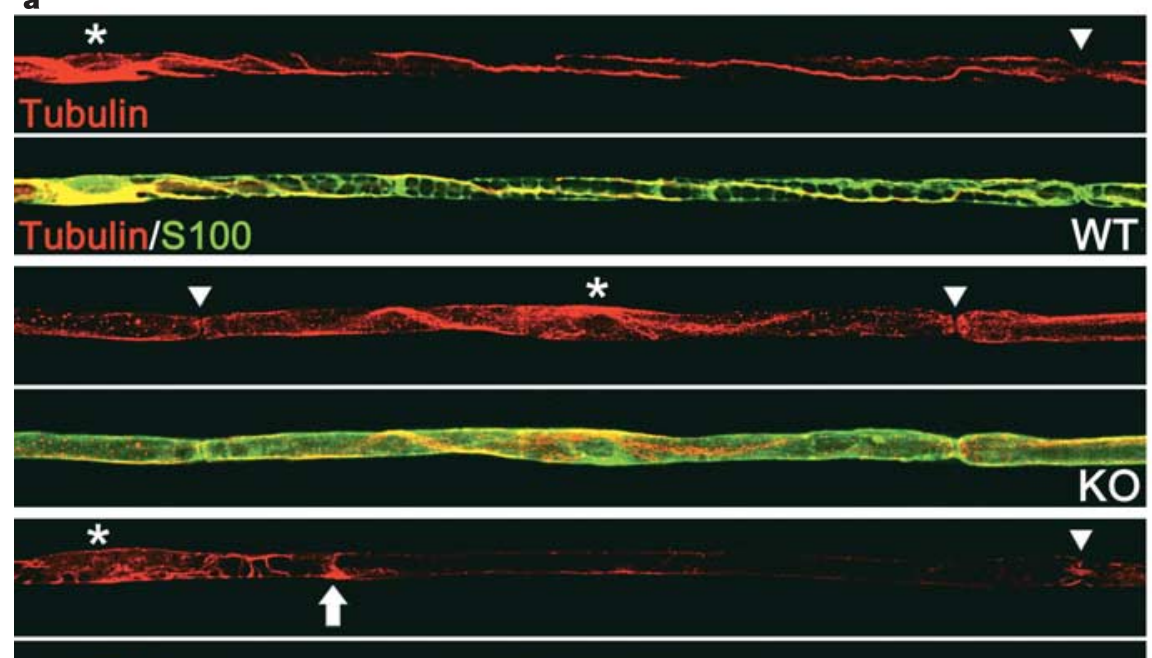

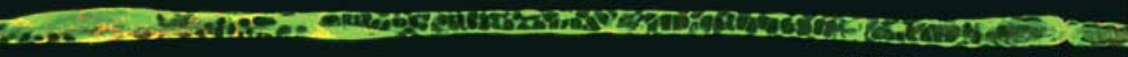
WT colchicine

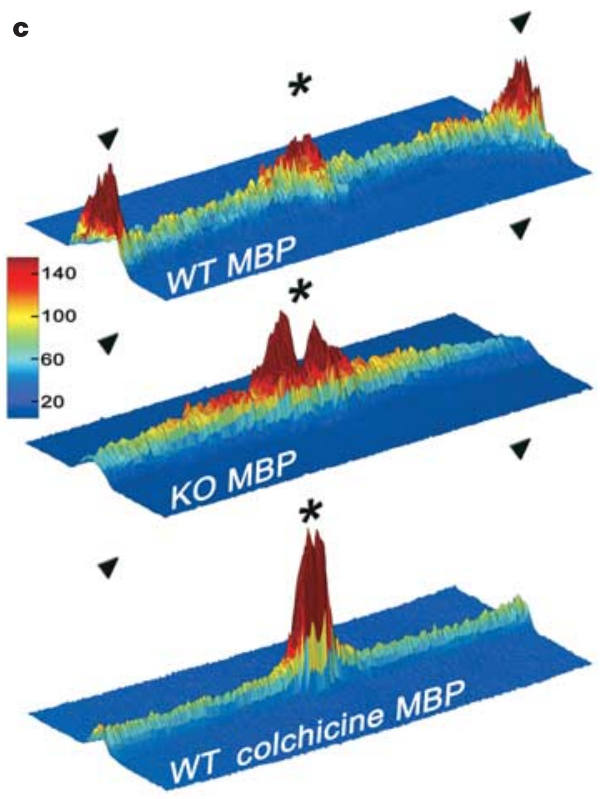

b

MBP d
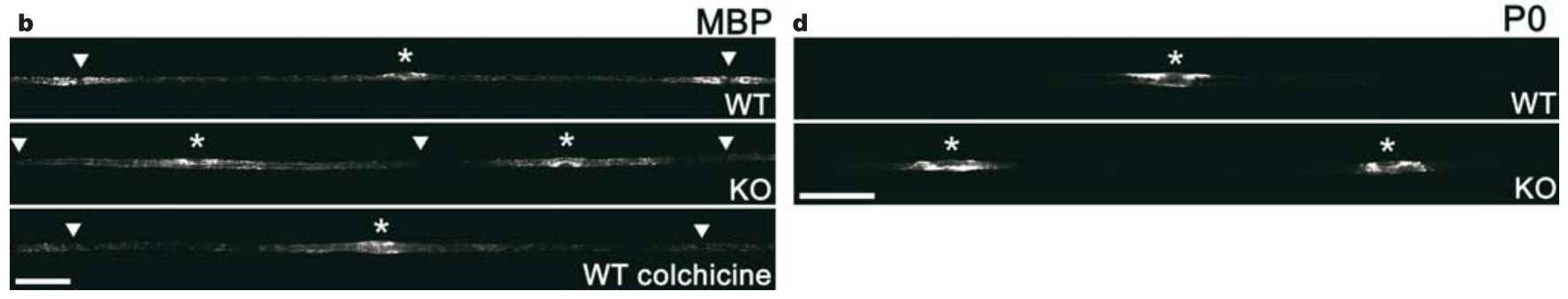

Figure 4 Intact Cajal bands and microtubules are required for MBP mRNA localization at the paranodes. Tubulin (red) and the Cajal band marker S100 (green) were visualized by immunofluorescence, and MBP and P0 mRNA were detected by in situ hybridization (images photographically inverted for clarity) in teased sciatic nerve fibres from 3-weekold WT and KO nerves with digoxigenin-labelled probes. a, In WT Schwann cells tubulin localizes with S100 in the Cajal bands, whereas in the absence of the bands tubulin staining becomes punctate as the microtubules approach the paranodes. Complete depolymerization of WT Schwann cell microtubules with colchicine in vivo followed by recovery for 3 days allows the microtubules to reform and partly extend (arrow indicates

extent of growth). Note that Cajal bands are unaffected. b, MBP mRNA in WT Schwann cells accumulates in the perinuclear and paranodal domains, whereas in KO Schwann cells lacking Cajal bands the strong MBP mRNA signal declines from the perinuclear region towards the paranodes. Depolymerization of microtubules in WT cells prevents the concentration of MBP mRNA at the paranodes. Scale bar, $50 \mu \mathrm{m}$. c. Pseudocolour surface plot of the mean internodal signal intensity (scale, 0-255) for MBP mRNA in WT $(n=9)$, $\mathrm{KO}(n=12)$ and colchicine-treated WT Schwann cells $(n=10)$. d, P0 mRNA is localized to the perinuclear region in both WT and KO Schwann cells. Nuclei and nodes of Ranvier are indicated by asterisks and arrowheads respectively. Scale bar, $50 \mu \mathrm{m}$.

demyelination in mature mice ${ }^{4}$, the myelin sheath of murine quadriceps nerve fibres was of normal thickness at 3 weeks as measured by the $g$-ratio (axon diameter/fibre diameter) (wild type (WT), $\left.0.62 \pm 0.01 ; \operatorname{Pr} x^{-l-}, 0.63 \pm 0.01\right)$, and the mean fibre diameter was unchanged (WT, $6.73 \pm 0.06 \mu \mathrm{m} ; \operatorname{Pr} x^{-1-}$, $6.67 \pm 0.07 \mu \mathrm{m})$. Furthermore, there seemed to be no derangements to the protein content, protein localization or ultrastructural organization of the nodal or paranodal apparatus at 3 weeks (Supplementary Fig. 1).

In spite of the apparently normal assembly of myelin by Schwann cells, but consistent with Ramón y Cajal's proposal, there was a striking decrease in their longitudinal growth (Fig. 2a). At postnatal-day 2 (P2), when myelinating Schwann cells have established a 1:1 relationship with segments of quadriceps nerve axons, WT and knockout (KO) Schwann cells were the same length (Fig. 2b). WT Schwann cells then elongated at the same rate as the growing nerve, whereas Periaxin-null Schwann cells had lower growth rates; this deficit persisted into adulthood in spite of the fact that WT and $\mathrm{KO}$ quadriceps nerves grew to the same length (Fig. 2b). Consistent with the absence of spontaneous firing in their quadriceps nerves, we never observed naked axonal segments in $\mathrm{KO}$ mice. Hence, during the early rapid growth phase of the nerve, extra Schwann cells must be needed to ensheath nerves in the mutant, although their origin remains to be determined. At later phases after

P21 it seems that KO Schwann cells can match the slower rates of nerve growth.

These results prompted us to use chimaeric mice to test whether the ability of Schwann cells to elongate in response to axonal growth was cell-autonomous. Strain S129-derived embryonic stem (ES) cells tagged with tau-green fluorescent protein $(\mathrm{GFP})^{5}$ were injected into blastocysts to track the fate of ES cells carrying WT Prx alleles. We had previously shown that the GFP fusion transgene is robustly expressed in myelinating Schwann cells. To confirm that these ES cells developed into Schwann cells with normal internodal lengths, we injected them into C57BL/6 WT as well as KO blastocysts. In each case, peripheral nerves contained Schwann cells derived from both endogenous and injected stem cells (Fig. 3a). In contrast to the normal elongation of Schwann cells tagged with GFP in a WT or KO environment, KO Schwann cells were unable to elongate normally whether alone or flanked by GFP-tagged Schwann cells with normal longitudinal bands and WT internodal lengths (Fig. 3b). This indicated that the capacity to elongate was a cell-autonomous property of Schwann cells. Internodal lengths in chimaeric nerves containing both GFP-tagged Schwann cells (derived from strain S129 mice) and WT Schwann cells (from C57BL/6 mice) were greater than in WT C57BL/6 mice because these chimaeric mice were larger and had longer quadriceps nerves (compare Figs $2 \mathrm{~b}$ and 3b). In contrast, the internodal lengths of Periaxin-null Schwann 

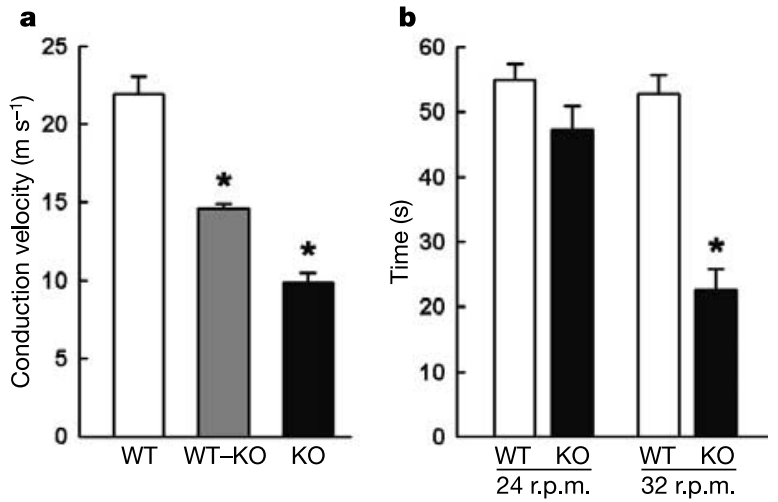

Figure 5 Peripheral nerve function is compromised in $\mathrm{PrX}^{-1-}$ animals. a, Nerve conduction velocities in 3-week-old quadriceps nerves were decreased in $\mathrm{KO}$ compared with WT mice, and chimaeras had intermediate values $(57.1 \pm 3.1 \%$ KO Schwann cells, $n=3$; mean \pm s.e.m.). Error bars indicate standard error of the regression coefficient (asterisks, $P<0.0001$ for comparisons with WT, Student's $t$-test; $n=4$ for WT and KO, $n=3$ for chimaeras). $\mathbf{b}$, A significant decrease in $\mathrm{KO}$ motor coordination compared with

cells in a chimaeric or pure C57BL/6 environment were indistinguishable (Fig. 3b). This further indicated that there was an upper limit to the ability of the mutant Schwann cells to elongate, irrespective of the extent of axon growth.

Microtubule-based messenger RNA translocation is believed to permit the incorporation of newly synthesized myelin basic protein (MBP) at the growing extremities of myelin-forming glia ${ }^{6-9}$. To test whether impaired transport of mRNA and proteins from the perinuclear to the peripheral regions of myelin-forming glia as a result of microtubule disruption might contribute to the decreased rate of elongation of Schwann cells lacking Cajal bands, we analysed the distribution of microtubules and MBP mRNA in single teased fibres from WT and KO mice. WT Schwann cells had an organized microtubule network that extended from the nucleus to the paranodes along the Cajal bands, whereas the tubulin staining in cells lacking Cajal bands frequently became punctate as the microtubules approached the paranodes (Fig. 4a). Importantly, the mutant Schwann cells could no longer accumulate MBP mRNA in the paranodal regions (Fig. 4b, c). Furthermore, the distal accumulation of MBP mRNA in WT Schwann cells was prevented when microtubules were reversibly disrupted, as found previously for oligodendrocytes $^{7}$ (Fig. 4a-c). Hence, deficits in microtubule-based transport might underlie the decreased capacity of Periaxin-null Schwann cells to elongate. In contrast to MBP mRNA, no discernible differences between WT and KO mice were observed in the distribution of the mRNA encoding the integral membrane protein P0 (Fig. 4d).

The decreased internodal length in KO mice, taken together with their normal axon diameter and myelin thickness, provided a unique opportunity to determine whether nerve conduction velocities are modulated by the internodal distance, as predicted from theory ${ }^{1}$. We measured the conduction time of compound action potentials in quadriceps nerves from 3-week-old WT, mutant and chimaeric mice. Nerve conduction velocities were decreased by more than $50 \%$ in KO compared with WT animals, and nerves that contained a mixture of WT and mutant Schwann cells had intermediate values, which was consistent with the effect of internodal length on conduction velocity (Fig. 5a). The substantial decrease in motor nerve conduction velocity in nerves lacking Cajal bands was accompanied by poorer performance on the RotaRod, a robust behavioural test for motor dysfunction (Fig. 5b). In sensory reflex behavioural tests, 3 -week-old mice did not display the neuropathic pain behaviour that results from demyelination in mature $\operatorname{Pr} x^{-1-}$ mice $^{4}$. They had normal withdrawal latencies in tests for both c

\begin{tabular}{|c|c|c|}
\hline Response & WT & KO \\
\hline $\begin{array}{l}\text { Withdrawal latency from noxious } \\
\text { heat (s) }\end{array}$ & $8.5 \pm 0.6$ & $8.4 \pm 0.5$ \\
\hline $\begin{array}{l}\text { Mechanical withdrawal threshold } \\
\left(\mathrm{mN} \mathrm{mm}{ }^{-2}\right)\end{array}$ & $281.7 \pm 15.5$ & $290.8 \pm 14.3$ \\
\hline
\end{tabular}

WT at 3 weeks was observed on the RotaRod at 32 r.p.m. but not at 24 r.p.m. Values are means \pm s.e.m. (asterisk, $P<0.0001$, Student's $t$-test; $n=6$ for WT and K0). c, 3-week-old WT and KO mice showed no significant differences in a cutaneous reflex test of hindpaw withdrawal threshold to mechanical stimulation or in their withdrawal latency to thermal nociceptive sensitivity. Values are means \pm s.e.m. ( $n=6$ for WT and $\mathrm{KO})$.

thermal hyperalgesia and mechanical allodynia, confirming the functional intactness of the myelin sheath (Fig. 5c). The relationship between the absence of longitudinal bands, decreased internodal length and impaired conduction in Periaxin-null mice was underlined by an analysis of CMTX mice, which also display late-onset demyelination. The quadriceps nerves from 3-week-old CMTX mice have normal bands and their Schwann cell lengths and conduction velocities are not decreased in comparison with WT animals (ref. 10 and Supplementary Fig. 2).

The physiological relationships between fibre diameter, myelin thickness and conduction velocity are well established ${ }^{11}$. However, our understanding of how internodal length influences conduction velocity has been largely theoretical ${ }^{1,12}$. We have used a recent model of mammalian motor nerve fibres that includes detailed morphological and electrical parameters ${ }^{13}$. As found in earlier theoretical treatments ${ }^{1}$, we found a substantial decrease in the rate of conduction when changes in the internodal distance were modelled within our experimental range from $500 \mu \mathrm{m}$ down to $250 \mu \mathrm{m}$ (Supplementary Fig. 3). Nerve conduction velocities therefore become much more sensitive to changes in internodal lengths in the shorter ranges.

Ranvier speculated that internodal length and nerve elongation are related during body growth because internodes are longer in larger animals ${ }^{14}$. From our studies, the ability of myelinating Schwann cells to elongate during the postnatal development of peripheral nerves was cell-autonomous but matched the growth rate of axons precisely, thus supporting the proposal that passive stretching could account for the establishment of internodal lengths during nerve growth ${ }^{15,16}$. Schwann cells lacking longitudinal bands were unable to keep pace with axon growth, and the consequence of this decreased capacity for elongation was that the internodal distances were shorter and conduction velocities were slowed in mutant nerves. We conclude that Schwann cell elongation is an essential feature of the functional development of the vertebrate nervous system. Finally, we propose that the longitudinal cytoplasmic structures in Schwann cells be named Cajal bands to acknowledge both Ramón y Cajal's discovery and our growing understanding of their function.

\section{Methods}

\section{Materials and antibodies}

Primary antibodies and dyes for microscopy were used at the following dilutions and concentrations; rabbit anti-DRP2 (ref. 3), 1:200; rabbit anti-S100 (Sigma), 1:200; rabbit anti-neurofascin ${ }^{17}, 1: 1000$; guinea pig anti-Caspr ${ }^{17}, 1: 200$; mouse anti-dystroglycan ${ }^{3}$, 1:100; mouse anti-pan- $\mathrm{Na}^{+}$channel (gift from M. Rasband), 1:300; rat anti-tubulin 
(YL1/2; Serotec), 1:200; chicken anti-betaIV-spectrin ${ }^{18}$ (gift from M. Komada), 1:100; 4,6diamidino-2-phenylindole (DAPI; Sigma), $4 \mu \mathrm{g} \mathrm{ml}^{-1}$; tetramethylrhodamine $\beta$ isothiocyanate (TRITC)-phalloidin (Sigma), $50 \mathrm{ng} \mathrm{ml}^{-1}$. The secondary antibodies were fluorescein isothiocyanate (FITC)-conjugated anti-rabbit IgG, 1:200; TRITC-donkey anti-guinea-pig IgG, 1:200; FITC-donkey anti-chicken IgY, 1:100 (all from Jackson ImmunoResearch); FITC-goat anti-mouse IgG1, 1:200 (Southern Biotech); Alexa Fluor-goat anti-rabbit IgG, 1:100 (Molecular Probes). For western blotting ${ }^{19}$, primary antibodies were used at tenfold greater dilutions.

\section{Microscopy and morphometry}

Depolymerization of Schwann cell microtubules in sciatic nerve in vivo was performed by topical treatment with colchicine as described ${ }^{20}$, and microtubules were allowed to reform for 3 days. By 5 days after colchicine treatment the microtubules had reached the paranodes showing that microtubule disruption is completely reversible (data not shown). Teased fibres were prepared from nerves fixed for $1 \mathrm{~h}$ in $4 \%$ paraformaldehyde, $0.1 \mathrm{M}$ sodium phosphate buffer $\mathrm{pH} 7.3$, and washed in several changes of phosphate buffer. The lengths of quadriceps nerves were measured from spinal cord exit to muscle insertion point. Teased fibres from quadriceps nerves of WT and KO mice were stained with TRITCphalloidin and DAPI, and 100 internodes and the lengths of two quadriceps nerves were measured for each animal $(n=3)$. For chimaeras, 200 internodal distances were measured for each Schwann cell type $(n=3)$. Nerves were prepared for electron microscopy as described previously ${ }^{4}$. For measurement of $g$-ratios, micrographs of randomly selected fields of ultrathin transverse sections of quadriceps nerves from 3-week-old WT and KO mice ( $n=3$ for each group, 120 fibres per group) were scanned and analysed using $\mathrm{NIH}$ Image. The $g$-ratio was calculated and all results are shown as means \pm s.e.m. The best fit for the growth rates of internodal and nerve length was found (F-test; Prism 3.03) and superimposed on the measured values shown in Fig. 2b. The growth rate of WT internodal length fitted a rectangular hyperbola, whereas the growth rate of $\mathrm{KO}$ internodal length fitted a straight line (linear regression). The growth rates of WT and KO nerve length both fitted a rectangular hyperbola. Bright-field images of teased sciatic nerve fibres stained for $\mathrm{P} 0$ and MBP mRNA by in situ hybridization with digoxigenin-labelled probes ${ }^{21}$ were photographically inverted to compare the signal intensity between WT, KO and colchicine-treated WT Schwann cells. Sense probes gave negligible background staining. Images of internodes were normalized by rescaling to a length of 400 pixels and imported into MATLAB software as a two-dimensional matrix representing pixel intensity. A threedimensional array was created by concatenating individual images in the $Z$ dimension. From this array, the mean value of pixel intensity in the $Z$ dimension was calculated for each pixel, resulting in a two-dimensional matrix.

\section{Electrophysiology}

Quadriceps nerves from 3-week-old KO and WT mice were transferred from oxygenated Krebs solution to an isolated chamber containing an array of $\mathrm{Ag} / \mathrm{AgCl}$ electrodes with $1-\mathrm{mm}$ intervals and surrounded by liquid paraffin maintained at $37^{\circ} \mathrm{C}$ for periods no longer than $10 \mathrm{~min}$. The proximal end of the nerve was excited by a square wave $(0.1 \mathrm{~ms}$, $0.1-1.5 \mathrm{~V}$ ) and the conduction distance was varied from $2 \mathrm{~mm}$ to $7 \mathrm{~mm}$ by altering the stimulating electrode position. The voltage was adjusted to ensure exact duplication of the active population, and the compound action potential was viewed on a storage oscilloscope. Values were stored as digitized signals with the use of Chart software (MacLab System). Conduction times were measured as described ${ }^{4}$.

\section{Behavioural testing}

Mice were conditioned to the RotaRod 1 day before the trial, and the RotaRod test was terminated either when the mouse fell from the rod or at $60 \mathrm{~s}$. Four trials per mouse were performed, separated by at least $10 \mathrm{~min}$ to avoid exhaustion of the animal. The thresholds and times for hindpaw withdrawal in response to graded mechanical stimulation and thermal stimulus were performed as described ${ }^{4}$.

\section{Chimaeras}

Chimaeras were obtained by injecting the ES cell line E14Tg2aSc4TP6.3 expressing GFPtagged tau protein into WT or KO blastocysts ${ }^{5}$. For internodal length measurements in quadriceps nerves, chimaeras were selected with an approximately equal contribution from each Schwann cell type.

Received 9 May; accepted 13 July 2004; doi:10.1038/nature02841.

1. Brill, M. H., Waxman, S. G., Moore, J. W. \& Joyner, R. W. Conduction velocity and spike configuration in myelinated fibres: computed dependence on internode distance. J. Neurol. Neurosurg. Psychiatry $\mathbf{4 0}$, 769-774 (1977)

2. Ramón y Cajal, S. Histology (Bailliere, Tindall \& Cox, London, 1933).

3. Sherman, D. L., Fabrizi, C., Gillespie, C. S. \& Brophy, P. J. Specific disruption of a Schwann cell dystrophin-related protein complex in a demyelinating neuropathy. Neuron 30, 677-687 (2001).

4. Gillespie, C. S. et al. Peripheral demyelination and neuropathic pain behavior in periaxin-deficient mice. Neuron 26, 523-531 (2000).

Pratt, T., Sharp, L., Nichols, J., Price, D. J. \& Mason, J. O. Embryonic stem cells and transgenic mice ubiquitously expressing a tau-tagged green fluorescent protein. Dev. Biol. 228, 19-28 (2000).

6. Ainger, K. et al. Transport and localization elements in myelin basic protein mRNA. J. Cell Biol. 138, 1077-1087 (1997)

7. Carson, J. H., Worboys, K., Ainger, K. \& Barbarese, E. Translocation of myelin basic protein mRNA in oligodendrocytes requires microtubules and kinesin. Cell Motil. Cytoskeleton 38, 318-328 (1997).

8. Colman, D. R., Kreibich, G., Frey, A. B. \& Sabatini, D. D. Synthesis and incorporation of myelin polypeptides into CNS myelin. J. Cell Biol. 95, 598-608 (1982).

9. Griffiths, I. R. et al. Expression of myelin protein genes in Schwann cells. J. Neurocytol. 18, 345-352 (1989).
10. Anzini, P. et al. Structural abnormalities and deficient maintenance of peripheral nerve myelin in mice lacking the gap junction protein connexin 32. J. Neurosci. 17, 4545-4551 (1997).

11. Hursh, J. B. Conduction velocity and diameter of nerve fibers. Am. J. Physiol. 127, 131-139 (1939).

12. Huxley, A. F. \& Stampfli, R. Evidence for saltatory conduction in peripheral myelinated nerve fibres. J. Physiol. (Lond.) 108, 315-339 (1949).

13. McIntyre, C. C., Richardson, A. G. \& Grill, W. M. Modeling the excitability of mammalian nerve fibers: influence of afterpotentials on the recovery cycle. J. Neurophysiol. 87, 995-1006 (2002).

14. Ranvier, L. Des étranglements annulaires et des segments interannulaires chez les Raies et les Torpilles. C. R. Acad. Sci. 75, 1129-1132 (1872).

15. Hiscoe, H. B. Distribution of nodes and incisures in normal and regenerated nerve. Anat. Rec. 99, $447-475$ (1947).

16. Schlaepfer, W. W. \& Myers, F. K. Relationship of myelin internode elongation and growth in the rat sural nerve. J. Comp. Neurol. 147, 255-266 (1973).

17. Tait, S. et al. An oligodendrocyte cell adhesion molecule at the site of assembly of the paranodal axoglial junction. J. Cell Biol. 150, 657-666 (2000).

18. Komada, M. \& Soriano, P. $\beta$ IV-spectrin regulates sodium channel clustering through ankyrin-G at axon initial segments and nodes of Ranvier. J. Cell Biol. 156, 337-348 (2002).

19. Gillespie, C. S., Sherman, D. L., Blair, G. E. \& Brophy, P. J. Periaxin, a novel protein of myelinating Schwann cells with a possible role in axonal ensheathment. Neuron 12, 497-508 (1994).

20. Trapp, B. D. et al. Polarization of myelinating Schwann cell surface membranes: role of microtubules and the trans-Golgi network. J. Neurosci. 15, 1797-1807 (1995).

21. Collinson, J. M., Marshall, D., Gillespie, C. S. \& Brophy, P. J. Transient expression of neurofascin by oligodendrocytes at the onset of myelinogenesis: implications for mechanisms of axon-glial interaction. Glia 23, 11-23 (1998).

Supplementary Information accompanies the paper on www.nature.com/nature.

Acknowledgements We thank H. Anderson and L. Ferguson for assistance, B. Smith for technical support, S. Scherer for comments, and K. Willeke and T. Ott of Bonn University for the CMTX mice. Figure 1a is reproduced with the permission of the Cajal Institute, CSIC, Madrid, Spain, copyright inheritors of Santiago Ramón y Cajal. This work was supported by the Wellcome Trust.

Competing interests statement The authors declare that they have no competing financial interests.

Correspondence and requests for materials should be addressed to P.J.B. (peter.brophy@ed.ac.uk).

\section{Calcium transients in astrocyte endfeet cause cerebrovascular constrictions}

\section{Sean J. Mulligan \& Brian A. MacVicar}

Brain Research Centre, Department of Psychiatry, University of British Columbia, 2211 Wesbrook Mall, Vancouver, BC, Canada, V6T 2B5

Cerebral blood flow (CBF) is coupled to neuronal activity and is imaged in vivo to map brain activation ${ }^{1}$. CBF is also modified by afferent projection fibres that release vasoactive neurotransmitters $^{2,3}$ in the perivascular region, principally on the astrocyte endfeet $^{4,5}$ that outline cerebral blood vessels ${ }^{6}$. However, the role of astrocytes in the regulation of cerebrovascular tone remains uncertain. Here we determine the impact of intracellular $\mathrm{Ca}^{2+}$ concentrations $\left(\left[\mathrm{Ca}^{2+}\right]_{i}\right)$ in astrocytes on the diameter of small arterioles by using two-photon $\mathrm{Ca}^{2+}$ uncaging ${ }^{7,8}$ to increase $\left[\mathrm{Ca}^{2+}\right]_{\mathrm{i}}$. Vascular constrictions occurred when $\mathrm{Ca}^{2+}$ waves evoked by uncaging propagated into the astrocyte endfeet and caused large increases in $\left[\mathrm{Ca}^{2+}\right]_{i}$. The vasoactive neurotransmitter noradrenaline ${ }^{2,3}$ increased $\left[\mathrm{Ca}^{2+}\right]_{i}$ in the astrocyte endfeet, the peak of which preceded the onset of arteriole constriction. Depressing increases in astrocyte $\left[\mathrm{Ca}^{2+}\right]_{\mathrm{i}}$ with BAPTA inhibited the vascular constrictions in noradrenaline. We find that constrictions induced in the cerebrovasculature by increased $\left[\mathrm{Ca}^{2+}\right]_{\mathrm{i}}$ in astrocyte endfeet are generated through the phospholipase $\mathrm{A}_{2}$-arachidonic acid pathway and 20-hydroxyeicosatetraenoic acid production. Vasoconstriction by astrocytes is a previously unknown mechanism for the regulation of CBF. 